\title{
Slope lengths and generalized augmented links
}

\author{
Jessica S. Purcell
}

\begin{abstract}
In this paper, we determine geometric information on slope lengths of a large class of knots in the 3-sphere, based only on diagrammatical properties of the knots. In particular, we show such knots have meridian length strictly less than 4 , and we find infinitely many families with meridian length approaching 4 from below. Finally, we present an example to show that, in contrast to the case of the regular augmented link, longitude lengths of these knots cannot be determined by a function of the number of twist regions alone.
\end{abstract}

\section{Introduction}

Given a knot in the 3-sphere whose complement admits a complete hyperbolic structure, by Mostow-Prasad rigidity the hyperbolic structure is unique, and therefore a knot invariant. However, it seems to be a difficult problem to determine geometric properties of the hyperbolic structure based only on a diagram of the knot.

In a recent paper [13], we discussed geometric properties of generalized augmented links. These allowed us to determine geometric information on large classes of knots in the 3-sphere, including geometric information on highly non-alternating knots, based only on a diagram.

In this paper, we extend the discussion of the geometry to information on slope lengths. In particular, we show such knots have meridian length strictly less than 4 , and we find infinitely many families with meridian length approaching 4 from below. This is of interest, because to date there are no known examples of knots with meridian of length 4 or more, although Agol has found a family of knots whose meridian length approaches 4 from below [7]. This paper provides evidence that knots with meridian length less than 4 are extremely common. Finally, we show that, in contrast to the case of the regular augmented link, longitude lengths of these knots cannot be determined by a function of the number of twist regions alone. We do 
this by presenting examples of knots with arbitrarily many twist regions, but with longitude length bounded by 4 .

The examples of knots of this paper are of interest, as they give classes of diagrams for which geometric information is available, but which are highly non-alternating and have arbitrarily large volume.

\subsection{Meridians}

A slope is defined to be an isotopy class of simple closed curves on a torus boundary of a 3-manifold. When the manifold admits a hyperbolic structure, the slope inherits a length: the length of a geodesic representative of the slope in a horospherical torus about the boundary.

A knot in the 3-sphere has at least one short slope: the meridian of the knot. By the 6-theorem, proved independently by Agol [7] and Lackenby [10], the meridian has length strictly less than 6 .

Interestingly, to date no knot complements have been found with meridian length at least 4. Agol found a family of examples of knots with meridian length approaching 4 from below [7]. Agol's knots are described as Dehn fillings of components of the 2-fold branched cover over one component of the Borromean rings, and in fact, they form a subset of the knots of this paper, as we will see below. Adams, and Adams and co-authors have found upper bounds on meridian lengths of certain classes of knots. In particular, 2-bridge knots have meridian length less than 2 [6], the meridian length of an alternating knot is at most $3-6 / c$, where $c$ is the number of crossings of the knot, and more generally, the meridian of a knot has length less than $6-7 / c[1]$. However, this general bound becomes little better than that given by the 6 -theorem as $c$ increases.

In this paper, we provide evidence that 4 may be a better upper bound on meridian length than 6 . We do so by presenting a family of links, called generalized augmented links, from which any knot may be obtained by Dehn filling. We show that the slope on these links which corresponds to the meridian of the knot has length strictly less than 4. Since high Dehn filling yields a manifold geometrically close to the original (see [16]), for any $\epsilon>0$ we need only exclude a finite number of slopes for each link component, and the knot obtained by Dehn filling will have meridian length within $\epsilon$ of that of the generalized augmented link. Thus if we enumerate knots by Dehn fillings of generalized augmented links, "most" knots have meridian of length less than 4.

We call the links generalized augmented links because they generalize a class of links, called augmented links, that has been studied extensively 
in the past. It is well known that any knot can be obtained by Dehn filling a regular augmented link. Actually, it was shown independently by the author with Futer [8] and by Schoenfeld [15] that these regular augmented links have meridians of length exactly 2. Note this implies that if we restrict to augmented links and their Dehn fillings, we obtain an enumeration of knots for which "most" knots actually have meridian lengths approaching 2 .

Of course, the result of 2 is unsatisfactory for many reasons, which partially motivated this paper. First and foremost, 2 is much shorter than the current largest known meridian length, i.e., the examples of Agol approaching 4 [7]. Thus if we wish to determine an upper bound, or find examples with larger meridians, we cannot consider high Dehn fillings on regular augmented links.

Secondly, knots obtained by high Dehn fillings of regular augmented links are very closely related to alternating knots. Such knots are either alternating or only non-alternating between twist regions. That is, they are only non-alternating in very restricted ways. In fact, regular augmented links actually give a very nice way of enumerating alternating knots (see, for example, $[5,11])$. By the above results, any examples of knots with meridian of length greater than 4 will most likely come from classes of highly nonalternating knots.

To that end, we investigate highly non-alternating knots and their meridian lengths. We will see that Dehn fillings of generalized augmented links give a nice description of many highly non-alternating knots. In addition, we show that generalized augmented links fit in with known meridian bounds much better than regular augmented links, by using them to construct families of knots whose meridian length approaches 4 from below. Thus, to Agol's example of a family of knots with meridian length approaching 4 from below, we add infinitely many more examples of families of knots with meridian length approaching 4 from below.

\subsection{Longitudes}

In [8], we showed that the longitude of a regular augmented link was bounded below by the number of twist regions of a diagram (we review the definition of twist region in Section 2).

In this paper, we show that no such result holds for generalized twist regions and generalized augmented links, by presenting an example of hyperbolic knots with arbitrarily many twist regions, but longitude length bounded near 4. 


\subsection{Organization}

This paper is organized as follows. In Section 2, we define generalized augmented links and present some examples. In Section 3, we prove that the slope which corresponds to the meridian has length less than 4. In Section 4 , we give examples of families of knots with meridians approaching length 4 from below. Finally, in Section 5, we present examples of knots with arbitrarily many generalized twist regions, but longitude length bounded near 4.

\section{Generalized augmented links}

\subsection{Twisting}

In [5], Adams defined a notion of augmented alternating links and proved that these were hyperbolic. We will generalize his definition. We also generalize a related notion, that of twist region of a knot or link diagram.

Definition 2.1. A twist region of a diagram is a portion of a diagram in which two strands twist about each other maximally, as in figure 1(a).

More formally, a diagram of a knot is a 4-valent graph with over-under crossing information at each vertex. This graph divides the projection plane into complementary regions with some number of sides. A bigon is a region with just two sides. A twist region of a diagram is the portion of a diagram inside a simple closed curve which bounds a string of bigons arranged end to end. We require twist regions to be alternating, else we can reduce the number of crossings in the region, and we require them to be maximal, in the sense that there are no other bigons adjacent to either end of the string of bigons in a twist region. A single crossing adjacent to no bigons is also a twist region.

Definition 2.2. A generalized twist region of a diagram is a portion of the diagram in which $m$ strands twist about each other maximally, for $m \geq 2$. see figure $1(b)$.

(a)

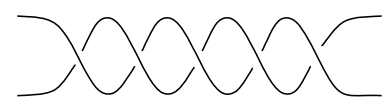

Figure 1: (a) A twist region. (b)

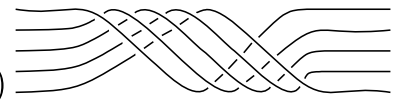

(b) A generalized twist region. 
More precisely, the two strands of a twist region bound an embedded ribbon surface. In a generalized twist region, additional strands are allowed to run parallel to the two strands, embedded on the ribbon surface.

In a regular twist region on two strands, a full twist consists of two crossings. Each strand twists once around the other and then exits the full twist in the same position in which it entered. A half twist is a single crossing of the two strands. We generalize these definitions to generalized twist regions.

Definition 2.3. A full twist in a generalized twist region is a region where the ribbon surface containing the strands of the twist region makes a full twist. That is, the two outermost strands of the generalized twist region form a regular twist region. In a full twist, those two strands twist around each other once. Figure 1(b) shows a single full twist of five strands.

Similarly, a half twist is a region where the ribbon surface of a generalized twist region makes a single half twist. This corresponds to a single crossing of the outermost strands, see figure 2 .

In figure 3, an example is shown of a portion of a diagram containing three twist regions. Notice it is highly non-alternating, and each twist region containing more than two strands is highly non-alternating. Do note that we could have selected each single crossing of this portion of the diagram to be a twist region on two strands. The choice of twist regions is not unique. However, that will not affect our results.

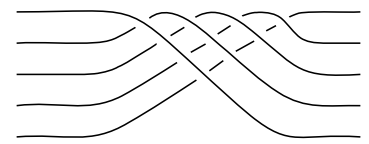

Figure 2: Five strands make a single half twist.

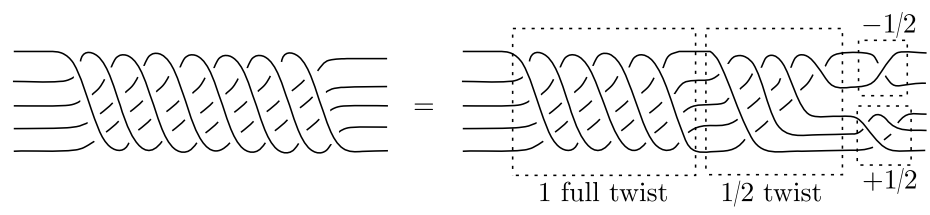

Figure 3: Five strands make a full and a half twist of all five strands, followed by a half twist of three strands and a half twist of two strands (in the opposite direction). 


\subsection{Augmenting}

We augment a diagram of a knot or link by inserting a simple closed curve encircling each generalized twist region. This is called a crossing circle, see figure 4. The components of the link coming from the original link components are called the knotting strands.

When each twist region has just two strands, as in the classical setting, the resulting link is called an augmented link. Provided a diagram is sufficiently reduced in obvious ways, an augmented link with at least two twist regions will be hyperbolic with very explicit geometry. See, for example, the papers of Futer and Purcell [8] and Purcell [14].

Definition 2.4. A generalized augmented link is a link which is formed from a knot or link diagram by augmenting each generalized twist region.

Generalized augmented links do not have quite as nice geometry as regular augmented links, but we can still deduce facts about their geometry.

Suppose in a knot or link diagram, the $i$ th twist region consists of $t_{i}$ full twists, plus possibly a half twist. We form the generalized augmented link $L^{\prime}$ by adding crossing circles. In particular, in the $i$ th twist region of the diagram of $L^{\prime}$, there are $t_{i}$ full twists. Obtain a new link $L$ by removing all $t_{i}$ full twists from the diagram of $L^{\prime}$, for each $i$. Thus $L$ has a diagram consisting of crossing circle components bounding the component from the knot. This knot component is either embedded in the projection plane, if all the $t_{i}$ happened to be even, or it may contain single half twists at crossing circles. The links of figure 5 are of this form.

Note $S^{3}-L^{\prime}$ and $S^{3}-L$ are homeomorphic as manifolds. This is because twisting and untwisting along a crossing circle $C_{i}$ give homeomorphisms of the solid torus $S^{3}-C_{i}$. If one of $S^{3}-L$ or $S^{3}-L^{\prime}$ is hyperbolic, MostowPrasad rigidity implies that the two manifolds are isometric. Thus we will assume from now on that a generalized augmented link has a diagram with all full twists removed from each generalized twist region.

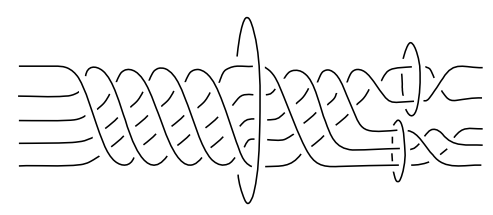

Figure 4: Insert crossing circles into knot diagram at each twist region. 

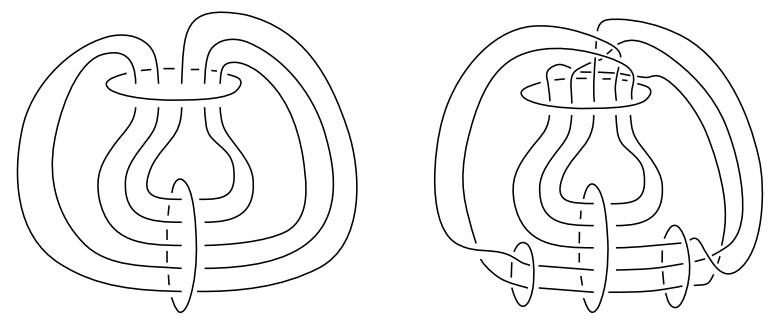

Figure 5: Untwisted generalized augmented links.

Note next that we may obtain the original knot complement by Dehn filling $S^{3}-L$ along crossing circles. In particular, if $t_{i}$ full twists were removed from the $i$ th twist region to go from the diagram of $L^{\prime}$ to the diagram of $L$, then by performing $1 / t_{i}$ Dehn filling on the $i$ th crossing circle, for each $i$, we obtain again the original knot complement. Consequently, any knot is obtained by Dehn filling a generalized augmented link.

\section{Meridians}

\subsection{Reflection and totally geodesic surfaces}

The key to the meridian slope length estimates for a hyperbolic generalized augmented link is the existence of embedded totally geodesic surfaces in the link complement.

Proposition 3.1. Any generalized augmented link complement admits an involution fixing a (possibly disconnected) embedded surface pointwise.

Proof. First consider the case where the generalized augmented link has no half twists. That is, all link components that are not crossing circles are embedded on the projection plane, as in the figure on the left in figure 5 . Then there is an involution of the link complement fixing the projection plane pointwise, obtained by reflecting the projection plane.

When there are half twists, the picture is only slightly more complicated. In this case, when we reflect in the projection plane, we obtain a new link in which all crossings of the half twists have been reversed. That is, they remain half twists, only now in the opposite direction of those in the original diagram. There is a homeomorphism taking the original link complement to that with half twists reversed: simply twist one full time around each 
crossing circle which contains half twists. Following the reflection with the inverse of this homeomorphism gives the desired involution.

Definition 3.2. The reflection surface refers to the surface fixed pointwise by the involution of Proposition 3.1.

When the generalized augmented link is hyperbolic, it is a well-known consequence of the proof of Mostow's theorem that the involution must be an isometry, and the reflection surface must be totally geodesic. From now on, we restrict to hyperbolic augmented links, and we consider the reflection surfaces as totally geodesic embedded surfaces in the link complement.

Note from the proof of Proposition 3.1 that the reflection surface is just the projection plane when the augmented link has no half twists. When the augmented link has half twists, the reflection surface still corresponds to the projection plane outside a neighborhood of the disks bounded by the crossing circles. We call these disks crossing disks. Notice also that the knotting strand link components are embedded in the reflection surface.

Lemma 3.3. Let $K$ be a knotting strand of a hyperbolic generalized augmented link. Then a meridian of $K$ is isotopic to a curve perpendicular to the reflection surface, which intersects it twice.

Proof. We may isotope the meridian to lie outside all neighborhoods of crossing disks. Away from these neighborhoods, the reflection surface is the projection plane. The projection plane divides $S^{3}$ into two balls, $S^{+}$and $S^{-}$. In a small neighborhood of a point on $K$, a meridian runs from the projection plane, up across the top of a tubular neighborhood of $K$ through $S^{+}$, then back to the projection plane. It then runs along the bottom of a tubular neighborhood of $K$ through $S^{-}$, closing again at the projection plane. Thus it meets the projection plane, hence the reflection surface, twice.

Note we can arrange the meridian to be taken to itself by the involution of $S^{3}-L$, with reversed orientation. Hence it must be orthogonal to the reflection surface.

\subsection{The universal cover}

To find bounds on the lengths of meridians, we will need to use the hyperbolic structure on $S^{3}-L$. Let $K$ denote the knotting strand of $L$. The 
length of a meridian will be measured on a maximal cusp about $K$. Recall that the maximal cusp is obtained by expanding a horoball neighborhood about the cusp until it meets itself.

Under the hyperbolic metric, the boundary of a maximal cusp is a horospherical torus, with an inherited Euclidean metric. We will find bounds on the length of the meridian of the torus under this metric.

Consider the upper half space model $\{(w, t) \mid w \in \mathbb{C}, t>0\}$ of the universal cover $\mathbb{H}^{3}$ of $S^{3}-L$. Conjugate such that a cusp lifts to the point at infinity.

The reflection surfaces, as totally geodesic surfaces which meet each cusp, will lift to totally geodesic planes in $\mathbb{H}^{3}$ which meet lifts of cusps. Thus in particular, there must be lifts of reflection surfaces to parallel vertical planes in $\mathbb{H}^{3}$.

The involution of $S^{3}-L$ lifts to a reflection of $\mathbb{H}^{3}$ through each vertical plane, whose projection under the covering map is an isometry. Thus the Euclidean distance between these lifts of reflection surfaces is constant.

Now consider the points of $\mathbb{C}$ on the boundary at infinity of $\mathbb{H}^{3}$ that project to cusps of $S^{3}-L$. Lifts of the geodesic reflection surfaces must also meet at each of these points. Thus at each such point we will see totally geodesic planes, which are Euclidean hemispheres. Their boundaries on $\mathbb{C}$ form a collection of tangent circles and lines. Because the reflection surfaces are embedded, the entire collection of lifts of reflection surfaces, including hemispheres and vertical planes in $\mathbb{H}^{3}$, will consist of disjoint planes, tangent only at points at infinity.

Finally, we add to this picture a collection of horospheres. A maximal cusp about the component $K$ of $S^{3}-L$ will lift to give a collection of horoballs in $\mathbb{H}^{3}$. The boundaries of these are horospheres, which are Euclidean spheres, tangent to points of the boundary at infinity that project to cusps. Because we are considering a maximal cusp, the horospheres will be tangent to each other in pairs.

By Lemma 3.3, a meridian of $K$ lifts to a curve which crosses two lifts of reflection surfaces orthogonally. Thus the length of a meridian must be exactly twice the Euclidean distance between lifts of two reflection surfaces, as measured on one of the horospheres about a point projecting to $K$. We will find bounds on this distance.

Theorem 3.4. A meridian of the knotting strand of a hyperbolic generalized augmented link, as measured on the maximal cusp about that component, has length strictly less than 4. 
Proof. Let $L$ be a hyperbolic generalized augmented link. Consider a crossing circle $C_{1}$ of $L$. This bounds a crossing disk $D$. The disk is punctured by the knotting strand $K$ a total of $m$ times, where $m$ is the number of strands of the generalized twist region, and $m \geq 2$. Note that a neighborhood of $K$ intersects $D$ in meridians of $K$. Note also that $D$ is taken to itself under the involution of $S^{3}-L$ of Proposition 3.1 and that $D$ intersects the reflection surface. Hence the reflection surface divides $D$ into two disks, an upper half $D^{+}$and a lower half $D^{-}$. Similarly, the reflection surface divides each of the meridians of $K$ on $D$ into two pieces, an upper half and a lower half. We will use the disk $D^{+}$to bound the length of the upper half of the meridian. Since the reflection is an isometry of $S^{3}-L$, this will also give a bound on the length of the lower half.

Consider the universal cover $\mathbb{H}^{3}$ of $S^{3}-L$. Conjugate such that infinity projects to the cusp corresponding to the crossing circle $C_{1}$. The disk $D^{+}$ also lifts to the universal cover. It will generally not be totally geodesic, but we may isotope the intersections of $D$ with the reflection surface to be geodesics. These are embedded in reflection surfaces, which are totally geodesic, so in the universal cover these intersections run across embedded totally geodesic planes. Since $D^{+}$meets $C_{1}$, the edges of $D^{+}$run from infinity, across a string of tangent geodesic planes which project to reflection surfaces, and back to infinity, see figure 6 .

Now, as described above, a maximal cusp about $K$ lifts to a collection of horoballs in $\mathbb{H}^{3}$. We will show that the distance between two lifts of reflection surfaces, as measured on the horospherical boundary of one such horoball, is strictly less than 2 . Since this length is one-half a meridian, it follows that the length of a meridian is strictly less than 4 .

Our proof is simplified by using an argument of Adams et al. [3]. In Theorem 4.1 of that paper, they show the width of a semifree geodesic surface is less than 2. That is, they show that if a manifold contains an embedded totally geodesic surface, if the surface meets the cusp exactly
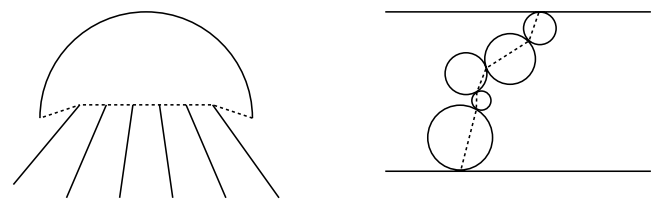

Figure 6: Left: dotted lines correspond to intersections of $D$ with the reflection surface. Right: in $\mathbb{H}^{3}$, these lift to geodesics running across lifts of the geodesic reflection surfaces. Note this is a view of $\mathbb{H}^{3}$ from infinity. There are also two dotted lines coming out from the horizontal lines to infinity. 
once, and if there is an embedded disk in the manifold whose edges lie on the geodesic surface, then the distance between lifts of the surface on a cusp in $\mathbb{H}^{3}$ is strictly less than 2. This is exactly the situation here, except that our geodesic surface, the reflection surface, meets the cusp more than once. However, because of the reflective symmetry in our case, the argument of Adams et al. will still apply. We reproduce it.

Consider the lift of the disk $D^{+}$to $\mathbb{H}^{3}$. In particular, consider its boundary. It consists of geodesics embedded on lifts of reflection surfaces, as in figure 6 , alternating with paths along horospheres at points of tangency of those planes. Thus we obtain a string of horospheres, each projecting to the horospherical torus about the maximal cusp of $K$. Let $H$ be the horosphere in this string of smallest Euclidean diameter. Consider the equator of $H$, that is, the circle on $H$ of largest diameter parallel to $\mathbb{C}$. We will show that the two hemispheres adjacent to $H$, which project to the reflection surfaces of $S^{3}-L$, intersect $H$ above its equator.

Suppose not. Suppose one of the hemispheres, say $S$, intersects $H$ below its equator. Then $S$ has boundary on $\mathbb{C}$ a circle of diameter strictly less than that of $H$. But then consider the edge of $D^{+}$which lies on $S$. This runs from $H$ at one end to another horosphere $H_{2}$ at the other end. Because the diameter of $S$ is less than that of $H$, the diameter of $H_{2}$ must be less than that of $H$, See figure 7 . This contradicts the fact that $H$ was the smallest.

Now, an arc along $H$ running from one lift of a reflection surface to another lies completely in the upper hemisphere of $H$. Hyperbolic geometry shows that the length of an arc running from a point on the equator to the opposite point on the equator has length exactly 2 . Thus the distance between reflection surfaces is at most 2 .

Suppose the distance is exactly 2. Consider again the string of horospheres and geodesics bounding the lift of $D^{+}$in the universal cover. The geodesics must meet the horospheres in their equators, which implies that

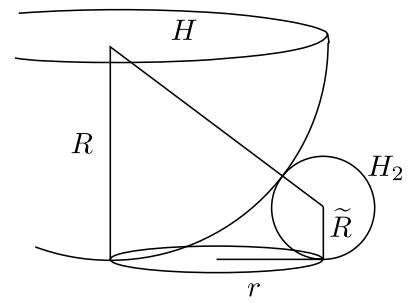

Figure 7: If $S$ intersects $H$ below its equator, then the radius $r$ of $S$ is strictly less than the radius $R$ of $H$. This implies that the radius $\widetilde{R}$ of $H_{2}$ must be strictly less than the radius $R$ of $H$. 
each horosphere is exactly the same height and all the horospheres are tangent. But then consider $B$, one of the horospheres lying under a geodesic of the sequence which runs to infinity. $\partial D^{+} \cap B$ runs between two reflection surfaces, so has length 2. But $\partial D^{+} \cap B$ is an arc which must run from the top of $B$ to its equator. This has length 1 , giving a contradiction.

Thus the distance between reflection surfaces is strictly less than 2. A meridian runs between two reflection surfaces, so its length is strictly less than 4.

\section{Families with meridians approaching 4}

In this section, we find generalized augmented links whose meridian length approaches 4 from below, showing that Theorem 3.4 is sharp.

The links we consider are links with two crossing circles. We define them as Dehn fillings of the link shown in figure 8, as described below. These links seem to have very interesting geometric properties in addition to the meridian lengths described here. In a forthcoming paper with Futer and Kalfagianni, we will analyze their volumes.

The link in figure 8 is a fully augmented 2-bridge link, with an extra reflective symmetry through the plane $P$ on which $L_{0}$ is embedded. $L_{0}$ is the link component which will become our knot.

Note that for $1 \leq i \leq n$, the link components $L_{i}$ and $L_{-i}$ bound an annulus in $S^{3}$. The Dehn fillings along slope $1 / r_{i}$ on $L_{i}$ and $-1 / r_{i}$ on $L_{-i}$ give a twist $r_{i}$ times about the annulus. This restricts to $r_{i}$ Dehn twists of the horizontal 4-punctured sphere $P$ on which $L_{0}$ is embedded, see figure 9 .

Note that if we perform these Dehn fillings in order, first on $L_{1}$ and $L_{-1}$, then $L_{2}$ and $L_{-2}$, etc., the result is a link consisting of two crossing circles,

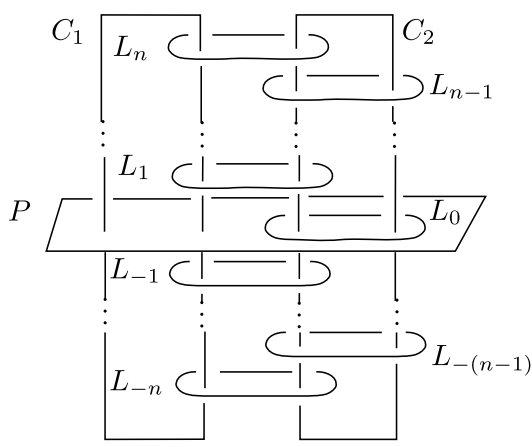

Figure 8: Dehn filling along $L_{i}$ and $L_{-i}$, with slope $1 / r_{i}$ and $-1 / r_{i}$, respectively, for $i=1, \ldots, n$, gives generalized augmented link. 

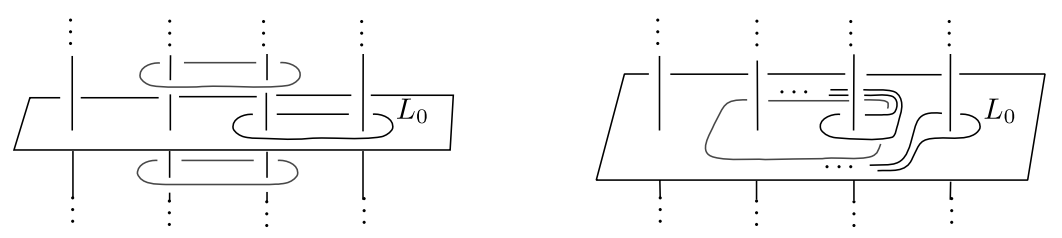

Figure 9: The upper and lower link components (left) bound an annulus. Dehn filling the top component along slope $1 / r_{i}$, and filling the bottom along slope $-1 / r_{i}$ yield $r_{i}$ Dehn twists of the 4 -punctured sphere (right).

$C_{1}$ and $C_{2}$, and a curve $K$ on the plane $P$, which is obtained by a sequence of Dehn twists applied to $L_{0}$. This is a generalized augmented link. In fact, the link on the left in figure 5 has this form.

The geometry of fully augmented 2-bridge links is very well understood. In particular, we showed in [14] that a fully augmented 2-bridge link decomposes into two identical, ideal, totally geodesic, right angled polyhedra. We will briefly explain the decomposition here. We refer the reader to $[14$, Section 6.4] for more details.

The two polyhedra are obtained by cutting the fully augmented 2-bridge link along the geodesic surface of the projection plane of figure 8. This divides the 3 -sphere into two pieces, one above and one below the projection plane. It divides each of the 2-punctured disks bounded by crossing circles into two pieces. Cut along each of these pieces, see figure 10(a).

This divides the manifold into two ideal polyhedra. The ideal vertices correspond to portions of the link, see figure 10(b). The faces come from the projection plane as well as from the 2-punctured disks. The faces are all totally geodesic, since the corresponding surfaces are geodesic in the augmented link. Thus when we give a polyhedron its hyperbolic structure,

(a)

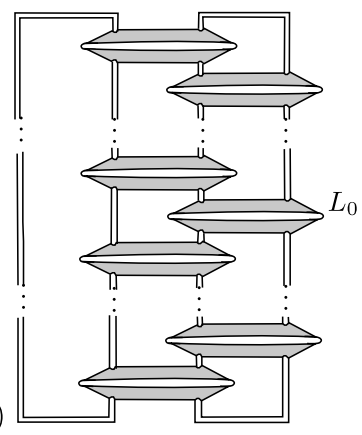

(b)

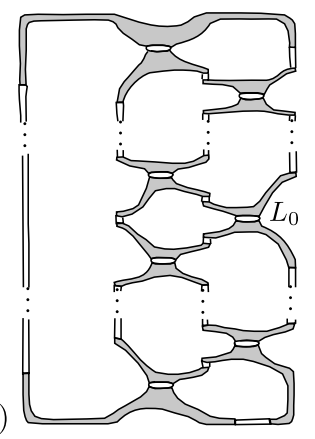

Figure 10: (a) Cut along the projection plane, and along disks bounded by crossing circles. (b) Retract neighborhood of the link to ideal vertices. 
(a)

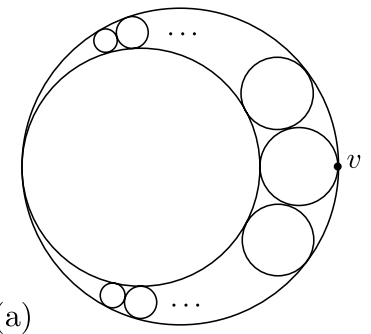

(b)

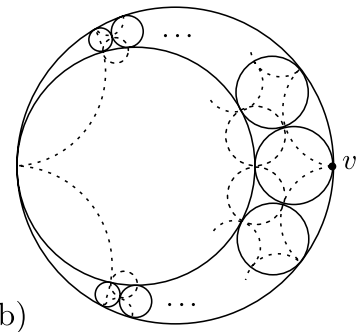

Figure 11: Circles giving polyhedral decomposition of augmented 2-bridge link.

faces correspond to (Euclidean) hemispheres in $\mathbb{H}^{3}$ orthogonal to the sphere at infinity.

One of the two polyhedra is given by the circle packing of figure 11(a). The circles should be viewed as boundaries of spheres on the plane at infinity of $\mathbb{H}^{3}$. They determine totally geodesic planes in $\mathbb{H}^{3}$. These are the geodesic planes from the projection plane. To these circles should be added additional circles, which meet the given circles exactly in points of tangency, as in figure $11(\mathrm{~b})$. These are from the 2-punctured disks. Together, these planes cut out an ideal geodesic polyhedron. To obtain the fully augmented link complement, we may glue two of these polyhedra together by gluing faces in a manner that reverses the cutting procedure described above.

We are interested in the cusp shape of the link component $L_{0}$, since after high Dehn filling, it will become the knotting strand of our generalized augmented link (with $C_{1}$ and $C_{2}$ crossing circles).

The vertex marked $v$ in the figure is the only ideal vertex of the polyhedron that projects to the cusp $L_{0}$. Thus the cusp shape of $L_{0}$ is obtained by determining the vertex shape of $v$, that is, the shape of a horosphere intersected with this ideal vertex of the polyhedron, and gluing two of these together: one for each of the two polyhedra.

Lemma 4.1. The shape of the cusp corresponding to $L_{0}$ is a square.

Proof. The vertex shape of $v$ is determined by taking the point $v$ of figure $11(\mathrm{a})$ to infinity under a Möbius transformation. The result is a collection of circles as in figure 12(a). Two of these glue together to form the cusp $L_{0}$, as in figure $12(\mathrm{~b})$. Note that the cusp shape of $L_{0}$ is a square, with meridian length $2 s$ and longitude $2 s$, where $s$ may be determined by finding the length of a side of the square in a maximal cusp.

It remains to determine the maximal cusp. 
(a)

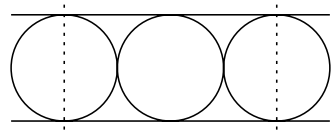

(b)

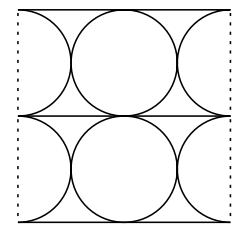

Figure 12: (a) The shape of the vertex corresponding to $v$. (b) The shape of the cusp corresponding to $L_{0}$.

Lemma 4.2. Under the metric on a maximal cusp, the meridian and longitude of $L_{0}$ both have length 4.

Proof. Since $v$ is the only vertex of the polyhedron that projects to the cusp $L_{0}$, we may expand a horoball about $v$ without it bumping any of its translates at least until the horoball becomes tangent to a face of the polyhedron.

Consider again figure $12(\mathrm{~b})$. Assume this picture is a view of $\mathbb{H}^{3}$ from infinity. We may also assume that three corners of the square lie at 0 , 2 and $2 i$ in $\mathbb{C}$, so that the circles of the figure have diameter 1 in the Euclidean metric on $\mathbb{C}$. Thus the corresponding hemispheres have Euclidean radius $1 / 2$. Then a horoball about infinity will not become tangent to one of these hemisphere faces until its boundary is at height $1 / 2$. Hence there is an embedded horoball of height $1 / 2$. If we measure the length of a meridian and longitude on the corresponding horosphere, the lengths will be 4. This implies that on a maximal cusp, the lengths are at least 4 .

Finally, one may see that this length corresponds to the length on the maximal cusp by noting that the longitude of $L_{0}$ bounds a 3 -punctured sphere. It is well known, due to Adams, that the maximal length of a curve along a 3 -punctured sphere is exactly 4 (see $[2,4]$ ).

Theorem 4.3. There exist generalized augmented links with meridian lengths approaching 4 from below.

Proof. For any $\epsilon>0$, and any link of the form in figure 8, there exist integers $r_{1}, \ldots, r_{n}$ such that the generalized augmented link obtained by $1 / r_{i}$ filling on $L_{i}$ and $-1 / r_{i}$ filling on $L_{-i}$ yields a generalized augmented link with meridian of length $4-\epsilon$. 
Corollary 4.4. For any $\epsilon>0$, there exist infinitely many knots with two generalized twist regions whose meridian lengths are greater than $4-\epsilon$.

Proof. The links of Theorem 4.3 have exactly two crossing circles $C_{1}$ and $C_{2}$. Find such a link with meridian length greater than $4-\epsilon / 2$. Then there exist integers $c_{1}$ and $c_{2}$ such that Dehn filling $C_{1}$ along slope $1 / c_{1}$ and Dehn filling $C_{2}$ along slope $1 / c_{2}$ yield a knot with meridian length at least $4-\epsilon$.

Remark 4.5. Agol's examples in [7] of knots with meridian length approaching 4 are actually a subset of the knots of Corollary 4.4. Agol's knots are obtained by Dehn fillings on links of the form of figure 8 in which $n=1$, see [7, Figure 7].

\section{Longitude length is independent of twist number}

With Futer in [8], we showed that the length of a longitude of a regular augmented link, i.e., one with just two strands per twist region, is bounded below by a linear function of the number of crossing circles.

In this section, we give an example to prove that similar simple lower bounds will not be possible in the case of generalized augmented links. In particular, we show there exists a family of generalized augmented links with arbitrarily many twist regions, but longitude length at most 4 .

The example is given by links illustrated in figure 13. In particular, we start with a link $L_{2}$ consisting of a knotting strand and two crossing circles, $C_{1}$ and $C_{2}$, as shown on the left of that figure. This link $L_{2}$ can also be seen as a 2-bridge link with an extra augmentation component, exactly as in the previous section, in which the three-component links after Dehn twisting along annuli were 2-bridge links with an extra augmentation component. That is, in the link $L_{2}, C_{1}$ and $C_{2}$ form the components of a 2-bridge link
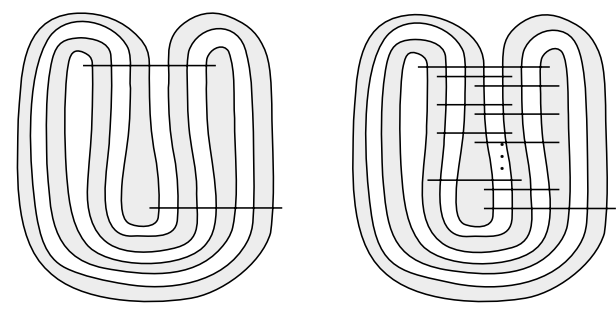

Figure 13: Left: the original link $L_{2}$ with two crossing circles. Crossing circles are represented by horizontal lines. Right: the generalized augmented link $L_{2 n}$ obtained by adding $n$ pairs of new crossing circles to $L_{2}$. 
with reflective symmetry. The knotting strand is an augmentation lying flat in the plane of the reflective symmetry. Since such 2-bridge links and their augmentations are known to be hyperbolic, $S^{3}-L_{2}$ must be hyperbolic. (This is a special case of a theorem on alternating knots, due originally to Menasco [12]. See also the paper of Futer and Gueritaud for a more direct proof [9] and Adams for the proof that augmentations are hyperbolic [5].)

Note the knotting strand and crossing circles divide the projection plane of the link $L_{2}$ into two distinct 3-punctured spheres. One of these 3-punctured spheres is shaded in figure 13. To form the new link $L_{2 n}$, add $n$ pairs of crossing circles as shown on the right of figure 13. These crossing circles are chosen so that their intersection with the projection plane is in the same 3-punctured sphere. (In the figure, their endpoints are in the shaded 3-punctured sphere.) Note the link is a generalized augmented link, with reflective symmetry through the projection plane.

Lemma 5.1. The generalized augmented link $L_{2 n}$ formed as above is hyperbolic.

We will prove this lemma at the end of the section. First, given this lemma, the results on longitude length are immediate.

Proposition 5.2. The length of the longitude of the knotting strand component of $S^{3}-L_{2 n}$ is at most 4 .

Proof. A longitude bounds a 3-punctured sphere on one side (i.e., the unshaded portion of the projection plane in figure 13). It is well known, due to Adams, that the length along a maximal cusp of a hyperbolic 3punctured sphere is at most 4 (see $[2,4]$ ). Hence the longitude length is at most 4 .

Proposition 5.2 shows that there can be no relationship between longitude length and number of generalized twist regions in a knot diagram.

It remains to show that $S^{3}-L_{2 n}$ is hyperbolic, that is, to prove Lemma 5.1. To do so, we show that $S^{3}-L_{2 n}$ is irreducible, boundary irreducible, atoroidal, with no essential annuli. Hyperbolicity then follows from work of Thurston [17].

Before we begin the proofs, note that we can decompose $S^{3}-L_{2 n}$ in a simple way. In particular, if we cut along the projection plane and the crossing disks (i.e., the disks bounded by the crossing circles), the result is (topologically) two 3-balls. Thus these surfaces chop the manifold $S^{3}-L_{2 n}$, 
and any submanifolds, into nice pieces. We use this fact repeatedly in the lemmas below.

Therefore, to simplify notation, we will denote the projection plane of $S^{3}-L_{2 n}$ by $P$. Note it has two components, one of which is a 3-punctured sphere. The two crossing circles $C_{1}$ and $C_{2}$ of $L_{2}$ bound crossing disks $E_{1}$ and $E_{2}$, respectively. That is, $E_{1}$ is a disk in $S^{3}$ with boundary the longitude of $C_{1}$, and $E_{1}$ meets the knotting strand of $L_{2}$ seven times. Similarly for $E_{2}$. The newly added crossing circles are denoted $C_{3}, C_{4}, \ldots, C_{2 n}$. Denote the crossing disk bounded by $C_{j}$ by $D_{j}$, for $j=3, \ldots, 2 n$. Each $D_{j}$ has boundary a longitude of $C_{j}$ and meets the knotting strand of $L_{2 n}$ four times. With this notation, we are ready to begin.

To prove $S^{3}-L_{2 n}$ is irreducible and boundary irreducible, we first prove the following simpler lemma.

Lemma 5.3. There does not exist a disk in $S^{3}-L_{2 n}$ whose boundary is a longitude of a crossing circle.

Proof. There cannot exist a disk in $S^{3}-L_{2 n}$ whose boundary is a longitude of $C_{1}$ or $C_{2}$, for such a disk would be a boundary compressing disk of $S^{3}-L_{2}$. Since $S^{3}-L_{2}$ is hyperbolic, no such disk exists.

Suppose there exists a disk $D$ whose boundary is a longitude of $C_{j}$, some $j \geq 3$. Consider the intersections of $D$ with the disks $E_{1}$ and $E_{2}$. If $D \cap\left(E_{1} \cup E_{2}\right)$ is non-empty, then some curve of the intersection bounds a subdisk $E$ in $D$. We may assume $E$ meets the projection plane, for otherwise $\partial E$ bounds a subdisk of $E_{1}$ or $E_{2}$ which meets no knotting strands, hence is embedded in $S^{3}-L_{2 n}$. We may therefore isotope off, decreasing the number of components of $D \cap\left(E_{1} \cup E_{2}\right)$. So assume $E$ meets $P$.

Now, after isotopy reducing intersections bounding disks, $E \cap P$ consists of an arc running from $E_{i}$ back to itself. Since each arc of the knotting strand runs from $E_{1}$ to $E_{2}$, no knotting strand is bounded between this arc and $E_{i}$. So $\partial E$ on $E_{i}$ bounds a disk which does not meet any knotting strands. Thus it bounds a disk on $E_{i}$, which is embedded in $S^{3}-L_{2 n}$. Again we may use this disk to push the intersection off $E_{i}$, decreasing the number of components of $D \cap\left(E_{1} \cup E_{2}\right)$.

Repeating this process, we may assume $D$ does not meet either $E_{i}$. But the intersection of $D$ with the projection plane is then an arc connecting the two points of intersection of the crossing circle $C_{j}$ with the projection plane, which does not run through either $C_{1}$ or $C_{2}$. This is impossible. 
Lemma 5.4. $S^{3}-L_{2 n}$ is irreducible.

Proof. Suppose $S$ is a 2-sphere in $S^{3}-L_{2 n} \subset S^{3}-L_{2}$. Because $S^{3}-L_{2}$ is irreducible, $S$ must bound a ball $B$ in $S^{3}-L_{2}$. If $S$ does not bound a ball in $S^{3}-L_{2 n}$, then one of the components $C_{j}$ must be contained in $B$. But $C_{3} \cup \cdots \cup C_{2 n}$ is a trivial link in $S^{3}$, so $C_{j}$ must be homotopically trivial in $S^{3}-L_{2 n}$. By the loop theorem, it bounds a disk whose boundary is a longitude of $\partial N\left(C_{j}\right)$. This contradicts Lemma 5.3.

Lemma 5.5. $S^{3}-L_{2 n}$ is boundary irreducible.

Proof. Suppose $D$ is a compressing disk for the boundary of $S^{3}-L_{2 n}$. By Lemma 5.3, $\partial D$ is not a longitude of a crossing circle. Since we are in $S^{3}, \partial D$ must be homotopically trivial on $\partial N\left(L_{2 n}\right)$, and we may assume it bounds a disk $E$ on this boundary surface. Then $E \cup D$ is a sphere $S$ in $S^{3}-L_{2 n}$. By Lemma 5.4, $S$ must bound a ball. But then we may isotope $D$ through this ball to lie on $E \subset \partial N\left(L_{2 n}\right)$, and $D$ is not a compressing disk.

Lemma 5.6. $S^{3}-L_{2 n}$ is atoroidal.

Proof. Suppose not. Let $T$ be an essential torus in $S^{3}-L_{2 n}$. We may assume first that $T$ meets the projection plane $P$, else it is an essential torus in one of the two handlebodies of $S^{3}-L_{2 n}-P$. No such torus exists.

Then by the equivariant torus theorem, we may assume that $T$ is preserved under the reflection in the projection plane.

We obtain a cell decomposition of $T$ by letting edges be intersections of $T$ with the projection plane, $T \cap P$, and intersections of $T$ with the crossing disks $T \cap E_{1}, T \cap E_{2}$ and $T \cap D_{3}, \ldots, T \cap D_{2 n}$. We let vertices be intersections of $T$ with $P \cap E_{i}, i=1,2$, and $P \cap D_{j}, j=3, \ldots, 2 n$. The faces lie in the two balls $S^{3}-L_{2 n}-P-\left(\cup E_{i}\right)-\left(\cup D_{j}\right)$.

Note each vertex of $T$ is 4 -valent. Thus if we let $v$ denote the number of vertices, $e$ denote the number of edges, $2 e=4 v$, or $e=2 v$. Next note there are no bigon faces or triangle faces of $T$. Let $f$ denote the number of faces. Since each edge is adjacent to two faces, $2 e \geq 4 f$. It follows from the Euler characteristic that every face must be a quadrilateral.

Now, first suppose $T$ meets none of the crossing disks $D_{3}, D_{4}, \ldots, D_{2 n}$. $T$ is a torus in $S^{3}-L_{2}$, which is hyperbolic, so $T$ must be compressible or boundary parallel in that manifold. Consider the intersections of $T$ with the projection plane of $S^{3}-L_{2}$. Both components of the projection plane are 3-punctured spheres in $S^{3}-L_{2}$, so the possible curves of intersection of 
$T$ with the projection plane components are limited. If a curve of intersection is trivial in the 3-punctured sphere, it remains trivial when we remove $C_{3}, \ldots, C_{2 n}$, since $T$ does not meet the crossing disks bounded by these circles. Thus in this case $T$ will be compressible in $S^{3}-L_{2 n}$. So a curve of intersection must be non-trivial in the 3-punctured sphere. Then $T$ is boundary parallel in $S^{3}-L_{2}$. In fact, it must be parallel to $C_{1}$ or $C_{2}$, since it does not meet $D_{3}, \ldots, D_{2 n}$.

Since $T$ is essential in $S^{3}-L_{2 n}$, some $C_{j}, j=1, \ldots 2 n$, must lie inside the solid torus $V$ bounded by $T$ containing $C_{1}$ or $C_{2}$. But note $C_{1}$ and $C_{2}$ both have endpoints lying in distinct components of the projection plane, whereas $C_{j}, j=3, \ldots, 2 n$ have endpoints lying in the same component of the projection plane. Thus $C_{j}$ must lie in a ball inside $V$. This is a ball in $S^{3}$. $C_{j}$ cannot be homotopically trivial, by Lemma 5.3 , so it must link $C_{1}$ or $C_{2}$ inside this ball. But this is impossible: the $C_{j}, j=1, \ldots, 2 n$, are all unlinked in $S^{3}$.

So $T$ must meet a crossing disks $D_{i}$. Consider $T \cap D_{i}$. By equivariance of $T$, this consists of closed curves encircling some number of intersections of $D_{i}$ with the knotting strands. Since there are just four such intersections, the curve must encircle 1, 2, 3 or 4 of these intersections.

In the case $T \cap D_{i}$ encircles one knotting strand: Faces adjacent to the edges $T \cap D_{i}$ are quadrilaterals. Two edges must run from $D_{i}$ along the projection plane to the next crossing disk, where they are joined by one other edge. Each edge along the projection plane runs parallel to the knotting strand, thus in the next crossing disk we see that $T$ again encircles one knotting strand. Continue. It follows that $T$ is boundary parallel, parallel to the knotting strand.

In the case $T \cap D_{i}$ encircles three knotting strands: Some adjacent face cannot be a rectangle, for one of the edges running from $D_{i}$ runs to a crossing disk $D_{i+1}$, say, while the other runs to a different crossing disk. Thus the endpoints of these edges cannot be joined by a single edge. This is a contradiction.

In the case $T \cap D_{i}$ encircles four knotting strands, either $T$ is boundary parallel, parallel to $C_{i}$, or we run into the same issue as in the previous case: the two adjacent edges terminate on distinct crossing disks, so the adjacent face cannot be a quadrilateral.

In the case $T \cap D_{i}$ encircles two knotting strands, the torus $T$ must enclose the component of $P$ which is a 3 -punctured sphere in $S^{3}-L_{2 n}$. Adjacent faces to $D_{i}$ may be quadrilaterals initially. However, eventually we will find a face must be adjacent to the lower crossing disk $C_{2}$, with one edge running parallel to the knotting strand to terminate on one side of $E_{1}$ and the other running parallel to the knotting strand to terminate on the other 
side of $E_{1}$ in the same component of $P$ minus the knotting strands. When we connect the endpoints of these edges by an arc, and reflect through the projection plane, we find they bound a disk on $E_{1}$. Hence $T$ is compressible.

Lemma 5.7. There are no essential annuli in $S^{3}-L_{2 n}$.

Proof. This follows from Lemma 5.6, for assume $A$ is an essential annulus. If $A$ has boundary components on two distinct link components, take the boundary of a small embedded neighborhood of $A$ and of the two link components of $L_{2 n}$ on which $\partial A$ lies. This is a torus in $S^{3}-L_{2 n}$, hence is inessential. It cannot be boundary parallel, so is compressible. The compressing disk cannot be on the side of the torus containing $A$ and the two link components, hence is on the outside. Cut along this disk. The result is a sphere in $S^{3}-L_{2 n}$. By Lemma 5.4, it bounds a ball in $S^{3}-L_{2 n}$. Since the ball cannot be on the side of the sphere containing $A$ and the two link components, it must be on the other side. But then the manifold $S^{3}-L_{2 n}$ has just two boundary components. This is a contradiction.

If $A$ has boundary components on the same link component, then the boundary of a small neighborhood of $A$ and the link component gives two tori in $S^{3}-L_{2 n}$, neither of which may be essential by Lemma 5.6. If either is compressible, consider the boundary of the compressing disk $D$. $\partial D$ cannot meet $A$ in a closed curve, else $A$ is compressible. Thus $\partial D$ meets $A$ in an arc. If the arc has both endpoints on the same component of $\partial A$, then a portion of the disk $D$ and a portion of $A$ together form a disk which must be isotopic to $\partial\left(S^{3}-L_{2 n}\right)$, by Lemma 5.5 , so we may slide this intersection off of $A$. Thus we assume the arc of intersection of $\partial D$ and $A$ is an essential $\operatorname{arc}$ on $A$. Then when we surger along $D$, we obtain a boundary compressing disk for $S^{3}-L_{2 n}$, hence by Lemma 5.5 , the result bounds a ball and so $A$ is isotopic to $\partial\left(S^{3}-L_{2 n}\right)$.

Thus when we take the boundary of a small regular neighborhood of $A$ and the link component on which $\partial A$ lies, the two resulting tori must be boundary parallel. But then there is some link component of $L_{2 n}$ lying inside. Then there exists an essential annulus with boundary components on this link component and on the link component on which the boundary of $A$ lies. We are then in the case of the first paragraph of this proof, and we have a contradiction.

\section{Acknowledgment}

The author is supported in part by NSF grant DMS-0704359. 


\section{References}

[1] C. Adams, A. Colestock, J. Fowler, W. Gillam and E. Katerman, Cusp size bounds from singular surfaces in hyperbolic 3-manifolds, Trans. Amer. Math. Soc. 358(2) (2006), 727-741 (electronic).

[2] C. Adams, Maximal cusps, collars, and systoles in hyperbolic surfaces, Indiana Univ. Math. J. 47(2) (1998), 419-437.

[3] C. Adams, H. Bennett, C. Davis, M. Jennings, J. Kloke, N. Perry and E. Schoenfeld, Totally geodesic Seifert surfaces in hyperbolic knot and link complements II, J. Diff. Geom. 79(1) (2008), 1-23.

[4] C.C. Adams, Thrice-punctured spheres in hyperbolic 3-manifolds, Trans. Amer. Math. Soc. 287(2) (1985), 645-656.

[5] —, Augmented alternating link complements are hyperbolic, Lowdimensional topology and Kleinian groups (Coventry/Durham, 1984), London Math. Soc. Lecture Note Ser., Vol. 112, Cambridge Univ. Press, Cambridge, 1986, 115-130.

[6] - Hyperbolic 3-manifolds with two generators, Comm. Anal. Geom. 4(1-2) (1996), 181-206.

[7] I. Agol, Bounds on exceptional Dehn filling, Geom. Topol. 4 (2000), 431-449.

[8] D. Futer and J.S. Purcell, Links with no exceptional surgeries, Comment. Math. Helv. 82(3) (2007), 629-664.

[9] F. Guéritaud, On canonical triangulations of once-punctured torus bundles and two-bridge link complements, Geom. Topol. 10 (2006), 12391284 (electronic), With an appendix by David Futer.

[10] M. Lackenby, Word hyperbolic Dehn surgery, Invent. Math. 140(2) (2000), 243-282.

[11] - The volume of hyperbolic alternating link complements, Proc. London Math. Soc. (3) 88(1) (2004), 204-224, With an appendix by Ian Agol and Dylan Thurston.

[12] W. Menasco, Closed incompressible surfaces in alternating knot and link complements, Topology 23(1) (1984), 37-44.

[13] J.S. Purcell, Augmenting, reflecting, and twisting: geometry of link complements in 3-manifolds, arXiv:math.GT/arXiv:0709.2919. 
[14] — Cusp shapes under cone deformation, J. Diff. Geom., to appear, arXiv:math.GT/0410233.

[15] E. Schoenfeld, Augmentations of knot and link complements, Undergraduate thesis, Williams College, 2003.

[16] W.P. Thurston, The geometry and topology of three-manifolds, Princeton Univ. Math. Dept. Notes, 1979.

[17] - Three-dimensional manifolds, Kleinian groups and hyperbolic geometry, Bull. Amer. Math. Soc. (N.S.) 6(3) (1982), 357-381.

Mathematical Institute

24-29 St Giles'

OXFORD, OX1 3LB

$\mathrm{UK}$

Department of Mathematics

Brigham Young UnIVERSITY

Provo, UT 84602, USA

E-mail address: jpurcell@math.byu.edu

Received February 29, 2008 
